\title{
س
}

$>\mathrm{DE}$

$\stackrel{1=1}{\simeq}$ PÉDAGOGIE

\author{
Recherches en éducation
}

174 | janvier-mars 2011

Évaluation, développement professionnel et

organisation scolaire

\section{Finalités socio-éducatives de la culture scientifique}

Socio-educational purpose of scientific literacy

Objetivos socioeducativos de la cultura científica

Sozio-erzieherische Finalitäten der wissenschaftlichen Kultur

Virginie Albe

\section{(2) OpenEdition}

1 Journals

Édition électronique

URL : http://journals.openedition.org/rfp/2789

DOI : $10.4000 / \mathrm{rfp} .2789$

ISSN : 2105-2913

Éditeur

ENS Éditions

Édition imprimée

Date de publication : 15 mars 2011

Pagination : 119-138

ISBN : 978-2-7342-1203-4

ISSN : 0556-7807

Référence électronique

Virginie Albe, "Finalités socio-éducatives de la culture scientifique», Revue française de pédagogie [En ligne], 174 | janvier-mars 2011, mis en ligne le 15 mars 2015, consulté le 10 décembre 2020. URL :

http://journals.openedition.org/rfp/2789; DOI : https://doi.org/10.4000/rfp.2789 


\section{NOTE DE SYNTHÈSE}

\section{Finalités socio-éducatives de la culture scientifique Virginie Albe}

La culture scientifique ou scientific literacy est devenue depuis plusieurs années un enjeu majeur de formation pour tous à l'échelle internationale, futurs scientifiques ou non. Une formation à la citoyenneté est ainsi visée dans le curriculum scientifique afin d'outiller tous les élèves à appréhender des problèmes impliquant des sciences dans leur future vie hors de l'école. Cette note de synthèse propose de questionner l'idée de développement de la culture scientifique en identifiant dans les nombreux discours et travaux sur ce thème les enjeux socio-éducatifs, les savoirs mobilisés et l'engagement social visé par les différentes approches de la culture scientifique.

Descripteurs (TESE) : réforme des programmes d'études, culture, sciences naturelles, compétence, finalités de l'éducation, savoir.

\section{INTRODUCTION}

Depuis plusieurs années, la formation du citoyen aux choix sociétaux est devenue un enjeu majeur de l'éducation scientifique pour de nombreux pays, notamment en Europe et dans les pays dits émergents (Albe, 2009 ; Robine, 2009). Une éducation aux sciences pour tous visant ce que d'aucuns nomment une culture scientifique et d'autres une alphabétisation ou une «littératie scientifique ", en traduction de la scientific literacy des anglophones, est alors largement promue dans les pays concernés (Albe, 2008). L'émergence de cette culture scientifique pour tous peut être reliée à une autre question majeure, largement (re)discutée à l'heure actuelle, sur l'éducation à la citoyenneté, dans le contexte de mondial isation de l'économie et en référence à un problème de cohésion des sociétés. Considérant que l'on observe une tendance des citoyens à un désengagement vis-à-vis de leur droit de regard sur les grands choix de société, symptôme apparent d'une dynamique de dépolitisation grandissante, la question cruciale de l'éducation à une citoyenneté active et éclairée émerge à nouveau. La contribution de l'éducation scientifique à la formation d'un citoyen que l'on souhaite actif et éclairé (question qui, en France, 
avait été un enjeu majeur de l'instruction primaire et de l'éducation populaire sous la Troisième République) est ainsi posée à nouveaux frais. Une réponse, ou la réponse adéquate pour ses promoteurs, résiderait dans le développement d'une culture scientifique et technique pour tous, et les arguments à l'appui de cette thèse sont souvent liés à une volonté de « repolitisation » des choix sociétaux dans lesquels les sciences sont impliquées. L'objet de cet article est de questionner l'émergence de cette nouvelle idée de culture scientifique, de repérer ses justifications dans les nombreux discours développés dans un contexte complexe de mutations sociales ${ }^{1}$ et enfin d'interroger les reconfigurations contemporaines de l'éducation scientifique dans la perspective d'une analyse sociopolitique du curriculum.

Le corpus élaboré pour cette note de synthèse a été constitué de la façon suivante : dans un premier temps, recherche de revues de littérature existantes sur le thème de science literacy ou scientific literacy en langue anglaise puis, dans un second temps, recherche de documents complémentaires publiés en langues française et anglaise avec les mots-clés " science literacy ", " scientific literacy » et " culture scientifique ", dans les revues majeures dédiées à la recherche en éducation aux sciences ${ }^{2}$ et ayant une large audience internationale : Journal of Research in Science Teaching et Science Education (éditées aux États-Unis), International Journal of Science Education et Journal of Curriculum Studies (éditées en GrandeBretagne), Revue canadienne de l'enseignement des sciences, des mathématiques et des technologies (éditée au Canada). Le corpus ainsi constitué comporte des documents de nature diverse : revues de littérature, rapports institutionnels et articles de recherche. Cinq revues de littérature ont été publiées en langue anglaise de 1983 à 2009, les plus récentes dans le Handbook of research on science education, qui fait le point sur l'avancée des recherches dans ce domaine (la dernière édition est de 2007, la précédente de 1998), et dans des revues de recherches en éducation aux sciences publiées en langue anglaise : le Journal of Research in Science Teaching, Studies in Science Education et Science Education (éditées aux ÉtatsUnis). Les sept rapports évoqués dans cette note de synthèse émanent d'associations (American Association for the Advancement of Science et Nuffield fondation, fondation britannique qui finance des projets et des recherches en éducation scientifique), d'organismes internationaux (OCDE), d'instances politiques (Commission européenne) et d'un ministère de l'Éducation (Canada). Ils ont été publiés de 1989 à 2008. Les 31 recherches empiriques et théoriques recueillies ont été publiées de 1958 à 2009.

\section{LA CULTURE SCIENTIFIQUE EN DÉBATS}

Plusieurs revues de littérature, dans le champ de l'éducation aux sciences, ont été consacrées à la culture scientifique, afin de clarifier ses orientations et définitions (Bybee, 1997 ; De Boer, 2000 ; Koballa, Kemp \& Evans, 1997 ; Mayer \& Kumano, 2002 ; Roberts, 2007). D'une approche historique effectuée sur une courte période récente et avec les précautions nécessaires étant donné le peu de recul que nous avons, il ressort que la culture scientifique constitue d'abord un slogan à la fin des années cinquante, pour faire ensuite l'objet de multiples définitions (Roberts, 2007). Le terme de "scientific literacy " apparaît initialement dans les écrits de Conant dès les années quarante (Holton, 1998 ; Bybee, McCrae \& Laurie, 2009) et se répand ensuite dans les discours d'éducateurs aux sciences (dans un champ professionnel, enseignants, formateurs et animateurs scientifiques dans les musées ou des ateliers scientifiques à l'école), en référence à un article de 1958 intitulé « Science literacy: 
Its meaning for American schools » (Hurd, 1958, cité dans Bybee, McCrae \& Laurie, 2009). Élaborer et mobiliser l'idée de culture scientifique est alors considéré comme un moyen de trouver du soutien dans le champ de l'éducation aux sciences afin d'examiner à nouveau les finalités de l'enseignement des sciences et élaborer des curriculums pour les « $90 \%$ d'étudiants [qui ne sont pas considérés comme des] scientifiques potentiels " (Roberts, 1983).

Ensuite, de la fin des années soixante à la fin des années soixante-dix, une série abondante et variée de définitions de cette expression est produite. Pour Roberts (2007), la scientific literacy est alors un concept parapluie, suffisamment large, au sens composite tel qu'il signifie tout et rien de particulier en éducation aux sciences. Le développement de la culture scientifique vise une compréhension large et fonctionnelle de la science dans un but d'éducation générale, et non pas de préparation des futurs scientifiques et technologues. Dans les années quatre-vingt, on assiste à un mouvement de mondialisation et s'affirme alors, de façon convergente, une volonté de développer la culture scientifique et technique. De telles préoccupations, sociales, économiques et environnementales, sont partagées par plusieurs pays et par des institutions internationales (en particulier, l'OCDE et l'UNESCO) ; elles conduisent des groupes de travail à proposer que la formation en sciences des futurs citoyens devienne une priorité, au même titre que la sélection et la formation des prochaines générations de scientifiques. La diffusion de la culture scientifique et technique pour tous, et pas seulement pour les élèves qui se destinent à des métiers scientifiques et techniques, est ainsi devenu un axe majeur des enseignements dans de nombreux pays. Cette perspective de développement de la culture scientifique soulève de nombreux débats chez les chercheurs. Certains y voient une possibilité de "réconcilier » élèves et citoyens avec les sciences et les technologies, d'autres considèrent que le développement de la culture scientifique constitue un nouveau slogan qui vise à rendre un enseignement de contenus scientifiques factuels plus attrayant, ou constitue l'expression nouvelle d'un vieux projet hégémonique d'occidentalisation du monde (Carter, 2005 ; Désautels \& Larochelle, 2003 ; Legendre, 2004). D'autres, enfin, y voient une possibilité de s'engager dans une refondation des programmes devenue nécessaire si l'on souhaite qu'ils permettent de former les citoyens de sociétés modernes, dans un contexte de mondialisation économique, d'augmentation de la production et d'expansion sans limites menaçant les libertés individuelles, le bien-être des sociétés et le futur de la planète (Hodson, 2003).

Les considérations sur ce qu'une personne cultivée en sciences peut être en mesure de faire sont très disparates et les orientations à privilégier font l'objet de grands désaccords (voir par exemple les débats dans les deux premiers numéros de la Revue canadienne de l'enseignement des sciences, des mathématiques et des technologies en 2002). II n'est donc pas possible de dégager de la littérature une définition consensuelle de scientific literacy ou science literacy; au contraire, c'est à un déluge de définitions (Roberts, 2007) que l'on se trouve confronté. Si le terme literacy est souvent pris comme une boîte noire dans le concept de scientific literacy ou de science literacy (employés comme synonymes), un sens fondamental minimal pourrait toutefois être dégagé de la littérature : lire et écrire en sciences. Mais la portée de la culture scientifique pour former en sciences et sa pertinence pour fonder un curriculum scientifique donnent lieu à de vifs débats.

La traduction de l'anglais vers le français suscite également des controverses. Alors que certains utilisent le terme d'« alphabétisation scientifique » qui, selon nous, met l'accent sur le sens fondamental de la "littératie " (lire et écrire), d'autres lui préfèrent celui de «culture scientifique » afin de désigner un sens plus complexe, ou de se référer à la tradition historique du contexte européen dans l'héritage philo- 
sophique du Siècle des lumières. Pour Solomon (1998) par exemple, le terme « culture » comporte une dimension élitiste qui le place à un niveau supérieur par rapport au terme "alphabétisation ". D'autres s'opposent à cette formulation de " culture scientifique », qui soulignerait le divorce entre sciences et humanités, pour des raisons historiques et de sélection et de formation, et appellent à inviter à nouveau la science au cœur de la culture humaine et humaniste (Robine, 2009). II ne m'appartient pas de trancher ce débat, la position que j'adopte ici consiste à suivre au plus près les termes employés dans les différents textes étudiés, et ainsi à reprendre souvent les termes anglais science literacy ou scientific literacy, voire à les traduire par «culture scientifique ». J'ajoute toutefois que la notion de culture me semble suffisamment fondamentale pour ne pas l'utiliser simplement comme une boîte noire, qu'elle invite à y regarder de plus près, ce qui engagerait de vastes débats en philosophie, anthropologie, histoire... bien loin du propos de ce texte. Ces réflexions orientent néanmoins la présente étude vers l'analyse de ce que les chercheurs en éducation aux sciences entendent par individu ou personne " scientifiquement cultivée ", notamment au regard de son engagement social, étant donné la justification rappelée en introduction de développer la culture scientifique pour pallier le désengagement politique des citoyens.

Mentionnons que tous ces débats ne nous apparaissent d'ailleurs pas surprenants dans la mesure où s'exprime, plus ou moins explicitement, à travers la volonté de développer la culture scientifique des jeunes, une vision de la finalité du curriculum scientifique. Ainsi on retrouve, à propos de la culture scientifique, une tension politique et intellectuelle continue inhérente au champ de l'éducation aux sciences entre visée de reproduction ou d'émancipation sociale, enseignement de contenus désocialisés ou acculturation à des pratiques sociales et culturelles. Approcher la littérature s'avère ainsi constituer un exercice difficile. Nous avons choisi de structurer cette note de synthèse à partir de l'identification, dans les nombreux discours et la grande diversité de positions sur la culture scientifique, des visées éducatives, de la nature des savoirs mobilisés et de l'importance accordée à l'engagement social et civique.

\section{VISÉES ÉDUCATIVES DE LA CULTURE SCIENTIFIQUE}

Différents types de culture scientifique, correspondant à des finalités éducatives diverses, ont été proposés dans la littérature. Pour Shen (1975) par exemple, une culture scientifique pratique consiste en la « possession de connaissances scientifiques qui peuvent être utilisées pour aider à résoudre des problèmes pratiques... [tels que] la santé ou la survie ${ }^{3}$ "(Shen, 1975, p. 46-47). Une culture scientifique civique est quant à elle destinée à " permettre au citoyen de devenir plus conscient des questions de sciences qu'il (ou son représentant) a à affronter et par conséquent le conduit à participer plus significativement aux procédés démocratiques dans une société de plus en plus technologique »(Shen, 1975, p. 48). Enfin, dans l'optique d'une scientific literacy culturelle, l'élève est " motivé par un désir de connaître les sciences comme une production humaine majeure. II s'agit de considérer les sciences comme on apprécie l'art » (Shen, 1975, p. 49).

Ces trois types de la scientific literacy ont par la suite été repris et hiérarchisés pour établir une progression dans le curriculum scientifique (Shamos, 1995). Elles ont aussi été étendues (Bybee, 1997), les différentes typologies étant discutées, retravaillées et saisies par des discussions polémiques. Pour Shamos (1995) par 
exemple, une scientific literacy (abrégée en SL par cet auteur) culturelle consiste en « la forme la plus simple de SL [...] une compilation d'informations d'un certain background que des communicants supposent que leur public possède déjà ». Un autre type de SL proposé par le même auteur, une scientific literacy fonctionnelle est telle que «l'individu non seulement possède un lexique scientifique, mais est aussi capable de discuter, lire et écrire de façon cohérente, en utilisant ces termes scientifiques dans un contexte qui n'est peut-être pas technique mais néanmoins signifiant » (Shamos, 1995, p. 88). Enfin Shamos propose un dernier type de SL, une scientific literacy « vraie » dans le sens où, pour lui, elle constitue un objectif approprié pour les étudiants qui se destinent à l'étude des sciences. Mais pour cet auteur, l'idée que la SL puisse orienter en général la finalité de l'éducation aux sciences est un mythe. II définit alors trois principes pour guider l'enseignement scientifique des étudiants non orientés en sciences, principes qui rejoignent les trois catégories proposées par Shen (1975) : “ À ce niveau, l'individu a des connaissances sur l'ensemble de l'entreprise scientifique [...] les cadres conceptuels majeurs [...] de la science, comment ils ont été élaborés, et pourquoi ils sont largement acceptés, comment la science produit de l'ordre à partir d'un univers aléatoire, et le rôle de l'expérience en science. L'individu apprécie aussi les éléments de l'investigation scientifique, l'importance d'un questionnement approprié, d'un raisonnement analytique et déductif, des procédés de pensée logique, et de confiance dans la preuve objective. » (Shen, 1975, p 89)

Pour Bybee (1997), la scientific and technological literacy est considérée comme un continuum dans lequel un individu développe plus ou moins une compréhension sophistiquée des sciences et des technologies. D'après lui, une « littératie nominale » implique que "l'individu associe des noms à un domaine général de sciences et de technologies [...] La relation [à] des définitions acceptables est réduite et insignifiante » (Bybee, 1997, p. 84). Bybee inclut dans ce type de SL les fausses conceptions, théories naïves et concepts imprécis. Ensuite, dans le cadre d'une « littératie fonctionnelle ", "l'individu répond de façon adéquate et appropriée au vocabulaire [...] il peut lire et écrire des passages avec du vocabulaire scientifique simple [...] II peut aussi associer le vocabulaire à des cadres conceptuels plus larges [...] mais éprouve de la difficulté à comprendre ces associations " (Bybee, 1997, p. 84-85). En revanche, par la maîtrise d'une " littératie conceptuelle et procédurale », " l'individu montre une compréhension à la fois des parties et de l'ensemble des sciences et technologies comme disciplines [...] II comprend la structure des disciplines et les procédures pour développer de nouvelles connaissances et techniques " (Bybee, 1997, p. 85). Un dernier type de SL, la « littératie multidimensionnelle », consiste en " la compréhension des structures conceptuelles essentielles des sciences et des technologies, ainsi que des caractéristiques qui rendent cette compréhension plus complète, comme par exemple l'histoire et la nature des sciences. En outre, l'individu à ce niveau comprend la relation des disciplines à l'ensemble des sciences et des technologies et à la société » (Bybee, 1997, p. 85).

Pour Fourez (1997), une personne « scientifiquement et techniquement cultivée » fait usage de ses connaissances pour son autonomie. Lorsqu'elle est confrontée à des contraintes naturelles ou sociales, ses connaissances lui donnent par exemple la possibilité de négocier ses décisions sans dépendance excessive à autrui, lui donnent également une capacité à communiquer et des moyens pratiques de faire face à des situations spécifiques. Pour Fourez, cette littératie ne porte pas seulement sur notre environnement matériel, mais aussi sur des situations émotionnelles, sociales, éthiques et culturelles. Elle ne concerne donc pas seulement les sciences de la matière et du vivant, mais aussi les sciences sociales. Ainsi le concept de 
culture scientifique et technologique renvoie pour Fourez à une émancipation de l'individu et non à l'acquisition de contenus ou capacités spécifiques.

Les arguments justifiant que les différents types de scientific literacy peuvent orienter l'éducation scientifique ont par ailleurs été catégorisés (Driver, Leach, Millar et al., 1996 ; Millar, 1996). Un argument économique consiste à mettre en avant le rôle des sciences et technologies dans l'insertion professionnelle. Un autre argument, qualifié d'utilitaire, consiste à considérer que, dans des sociétés technoscientifiques, les citoyens seront mieux en mesure d'affronter les problèmes s'ils ont une base de connaissances scientifiques et techniques. Selon un autre argument dit démocratique, il s'agit de viser une participation significative des citoyens aux décisions que les sociétés doivent prendre à l'égard de problèmes socioscientifiques et sociotechniques toujours plus complexes. Enfin un argument qui peut être qualifié de culturel envisage les sciences en tant qu'entreprise culturelle et sociale comme l'art, la musique et la littérature.

Par ailleurs, différentes définitions de la scientific literacy (ou culture scientifique ${ }^{4}$ ) ont été proposées dans le cadre des enquêtes internationales PISA (Program for International Student Assessment) en 2003 et 2006. Lors de l'enquête de 2003, la culture scientifique désigne la capacité d'utiliser des connaissances scientifiques, d'identifier des questions et d'élaborer des conclusions appuyées sur des preuves, afin de comprendre et d'aider à une prise de décision à propos du monde naturel et de ses changements dus à l'activité humaine (OCDE, 2006, p. 133). Trois procédés, considérés " centraux " dans cette culture scientifique, sont objets de l'enquête. II s'agit de :

- décrire, expliquer et prédire des phénomènes scientifiques ;

- comprendre l'investigation scientifique ;

- interpréter des preuves scientifiques et des conclusions.

Ces éléments ont été repris et complétés lors de l'enquête PISA 2006 par la considération des attitudes des jeunes envers les sciences et par l'accent mis sur la pertinence de connaissances sur les sciences (et pas seulement en sciences). La culture scientifique est alors considérée constituée de trois compétences clés (OCDE, 2006), vues comme fonctionnant en une séquence que des individus devraient suivre lorsqu'ils rencontrent et résolvent des problèmes liés aux sciences (Bybee, McCrae \& Laurie, 2009, p. 878). II s'agit d'identifier des questions scientifiques, puis d'expliquer scientifiquement des phénomènes et enfin d'utiliser des preuves scientifiques. On admet que ces trois compétences sont influencées par les savoirs scientifiques et les savoirs sur les sciences, ainsi que par les attitudes envers les sciences (intérêt, soutien à l'investigation scientifique, responsabilité vis-à-vis des ressources naturelles et de l'environnement). La notion de culture scientifique renvoie ici à l'acquisition et à l'application conséquente de savoirs et d'attitudes scientifiques et technologiques dans des contextes dits « de vie » (expression provenant directement de l'OCDE, 2006) pour un bénéfice personnel, social et global (Bybee, McCrae \& Laurie, 2009, p. 869). L'idée de contexte est majeure dans une telle définition et également dans l'évaluation des compétences des élèves en sciences lors de l'enquête PISA 2006. Nous détaillerons ces contextes lorsque nous explorerons la nature des savoirs mobilisés dans la culture scientifique. Tentons pour l'instant de cerner les finalités éducatives d'une telle approche de la culture scientifique. Viser chez les jeunes la capacité d'utiliser des savoirs scientifiques dans des contextes dits " de vie " impliquant des sciences nous semble relever d'une finalité pratique telle que proposée par Shen (1975) ou d'une finalité utilitaire selon la catégorisation de Millar (1996), faite à partir des arguments servant à orienter le curriculum scientifique sur les différents types de scientific literacy. 


\section{NATURE DES SAVOIRS MOBILISÉS DANS LA CULTURE SCIENTIFIQUE}

$\mathrm{Si}$, dans une perspective didactique, on examine la nature des savoirs mobilisés dans les situations d'apprentissage promues ou mises en œuvre dans des recherches empiriques en éducation aux sciences, plusieurs orientations peuvent être identifiées. S'agit-il de considérer l'éducation scientifique en fonction d'un projet de société ou en fonction des contenus des disciplines scientifiques ? Répondre à cette question trace une ligne de partage entre deux visions de la culture scientifique, conçues comme les deux extrêmes d'un continuum d'approches et identifiées à partir d'un vaste corpus de recherches et de textes institutionnels (rapports d'académies des sciences ou d'associations d'éducateurs en sciences, de l'UNESCO, de l'OCDE de 1973 à 20055, voir Roberts, 2007).

Dans une vision 1, il s'agit d'enseigner des contenus scientifiques pour euxmêmes (Roberts, 2007, p. 729). Les savoirs scolaires sont établis en référence à la science savante et considérés sans aucune implication dans la société (Arons, 1983 ; Snow, 1962). Cette conception de la culture scientifique correspond pour Laugksch (2000) à une « catégorie de l'apprendre 6 ». Dans une vision 2, les savoirs scolaires sont élaborés en référence à leur implication dans la société (Branscomb, 1981 ; Miller, 1983). Dans une telle approche, nommée " catégorie du fonctionnement en société 7 » par Laugksch (2000), la culture scientifique est un concept relatif et socialement défini qui diffère selon les périodes, les régions géographiques, les communautés ou les conditions sociales. II peut s'agir par exemple dans les programmes scolaires du choix de situations pour lesquelles la science semble légitimement jouer un rôle dans d'autres affaires humaines (Roberts, 2007, p. 729) ou de situations ayant une dimension scientifique et pouvant être rencontrées par les élèves en tant que citoyens (Roberts, 2007, p. 730). Cette centration sur les situations rejoint l'accent mis sur les contextes dits « de vie » dans l'enquête PISA 2006 et conduit certains de ses experts à revendiquer l'ancrage de PISA 2006 dans cette vision 2 de la culture scientifique (Bybee, McCrae \& Laurie, 2009, p. 866). Ces contextes « de vie » concernent la santé, les ressources naturelles, l'environnement, les risques environnementaux, technologiques et les frontières entre sciences et technologies (OCDE, 2006). II s'agit de les faire appréhender par les élèves aux niveaux local, social et global.

Pour prendre d'abord l'exemple de la santé, les élèves peuvent l'appréhender de la façon suivante : au niveau local, sa préservation, la prévention d'accidents et l'alimentation ; au niveau social, le suivi et les transmissions de maladies, la santé d'un groupe social et les choix alimentaires ; et au niveau global, les épidémies et la propagation de maladies infectieuses. Concernant les ressources naturelles, au niveau local, il s'agit de la consommation individuelle de matériaux et d'énergie; au niveau social, de la préservation de la qualité de vie (sécurité, production et distribution de nourriture et d'énergie) des populations humaines ; et au niveau global, des systèmes naturels renouvelables ou non renouvelables, de la croissance de la population mondiale, de l'usage durable des espèces. À propos de l'environnement, au niveau local, il s'agit d'un comportement respectueux de l'environnement et de l'usage des matériaux; au niveau social, de la répartition des populations, de la gestion de déchets, des impacts sur l'environnement et de la météorologie locale ; et au niveau global, de la biodiversité, de la durabilité écologique, du contrôle de la population, de la production et des sols. Les risques concernent, au niveau local, les décisions en matière de logement; au niveau social, les changements rapides (tremblements de terre, météorologie sévère), lents et progressifs (érosion côtière, sédimentation) et l'évaluation des risques ; et au niveau global, le changement climatique et l'impact de la guerre moderne. Concernant les frontières entre sciences et 
technologies, au niveau local il s'agit de l'intérêt pour les explications scientifiques des phénomènes naturels, des passe-temps basés sur les sciences, le sport et les loisirs, la musique et les technologies personnelles (ordinateurs, jeux vidéo, etc.) ; au niveau social, il s'agit des objets et services innovants, de la modification génétique, de la technologie de l'armement et du transport; et au niveau global, il est question de l'extinction des espèces, de l'exploration de l'espace et de l'origine et la structure de l'univers (OCDE, 2006).

Pour appréhender ces contextes dits " de vie » impliquant des sciences, il est attendu des jeunes qu'ils mobilisent des savoirs scientifiques et des savoirs sur l'investigation et les explications scientifiques. Un élève scientifiquement cultivé serait dans ce cas celui ou celle qui comprend des concepts scientifiques fondamentaux relatifs aux systèmes physiques (structure et propriétés de la matière, réactions chimiques, forces et mouvements, énergie et ses transformations, interactions entre énergie et matière), aux systèmes vivants (cellules, êtres humains, populations, écosystèmes, biosphère), à la Terre et à l'espace (structures, énergie et changements des systèmes terrestres, histoire de la Terre, gravité et système solaire) et aux systèmes technologiques (rôle des technologies basées sur les sciences, principes et concepts technologiques, relations entre sciences et technologies).

Dans les enseignements scientifiques pour le $\mathrm{xx} \mathrm{e}^{\mathrm{e}}$ siècle, élaborés et mis en œuvre en Angleterre dans une perspective de scientific literacy (Millar, 2006), de nouveaux contenus ont également été introduits dans le curriculum scientifique, comme l'épidémiologie ou la santé. L'accent est mis sur une compréhension large et qualitative des explications scientifiques et sur les connaissances sur les sciences. Ces deux visions de la culture scientifique comportent des risques sur le plan didactique et pédagogique. Pour la vision 1, il est possible d'imaginer l'introduction en classe, par un effet de la mode "culture scientifique " et comme source de motivation des élèves, de situations sur des thèmes où sciences et sociétés sont liées. Passée une telle introduction, c'est un enseignement scientifique traditionnel qui aurait lieu et la mission de développement de la culture scientifique des élèves serait considérée comme accomplie. La culture scientifique dans ce cas peut être vue comme un moyen de motiver les élèves pour l'apprentissage des sciences, comme un moyen de légitimer le curriculum scientifique dans l'enseignement secondaire obligatoire (Davies, 2004) ou comme un " supplément d'âme " une fois terminé l'enseignement des concepts scientifiques. Osborne (2007) souligne ainsi que les curriculums scientifiques contemporains sont essentiellement préparatoires à la formation de futurs scientifiques plutôt qu'à celle de futurs citoyens.

Dans la vision 2, le risque pourrait être, de façon opposée, de consacrer insuffisamment d'attention à la dimension scientifique des situations éducatives, et cela également par effet de mode, pour préférer par exemple des discours sur ou autour des sciences ou la mise en débat de questions sociales en contexte scolaire. L'expérience de curriculums "sciences technologies sociétés » (STS par la suite) développés dans les dernières décennies en Angleterre, au Canada, aux États-Unis et en Belgique notamment, peut être riche d'enseignements à ce propos. Dès le début des années soixante-dix émergent des recherches et des interventions éducatives sur des questions comportant des dimensions sociales, scientifiques et techniques en interdépendance. Bien qu'une telle perspective soit aujourd'hui considérée comme une contribution importante, sa portée dans les pratiques d'enseignement a bien souvent été considérablement diluée (Yager, 1996). D'une part, nombre de programmes STS ont été limités à l'incorporation de vignettes historiques ou sociales dans les programmes de sciences traditionnels (Bybee, 1991) : on retrouve ici le risque soulevé à propos de la vision 1 de la culture scientifique. D'autre 
part, des curriculums STS ont dû être abandonnés pour des raisons politiques, des contraintes bureaucratiques et sous l'influence d'éminentes personnalités scientifiques. Il est intéressant de se pencher ici sur quelques initiatives curriculaires pour comprendre les questions soulevées et pour mieux cerner les enjeux et débats liés aujourd'hui à la culture scientifique. Ainsi des curriculums STS pour l'enseignement scientifique dans le secondaire dans la province canadienne de l'Alberta, en remplacement des disciplines traditionnelles (biologie, chimie, physique), ont obtenu le soutien des éducateurs et des milieux politiques (Blades, 1997 ; Fensham, 1993 ; Gaskell, 1989). Lorsqu'il s'est agi de mettre au point de nouveaux manuels et des guides d'accompagnement de ces curriculums STS, de nombreuses oppositions ont été exprimées, par des professionnels et des scientifiques universitaires. Blades (1997) a relaté la manière dont professionnels et scientifiques universitaires se sont mobilisés pour "saboter » ces curriculums STS sur le plan politique. Leur argument majeur était que ces changements conduiraient à une diminution sévère du nombre d'ingénieurs, de géologues, de scientifiques et menaceraient à long terme le système de santé (selon l'Alberta Medical Association). Un comité de la faculté des sciences de l'Université de l'Alberta a également examiné les nouveaux programmes proposés et déclaré que « le résultat a abouti à des sciences sociales se faisant passer pour des sciences »(Blades, 1997, p. 61). Cela rejoint le risque soulevé à propos de la vision 2 de la culture scientifique. C'est alors sans surprise que les documents ont été profondément révisés, de façon à ce que la nouvelle discipline corresponde à un regroupement des contenus des trois disciplines traditionnelles.

Cette expérience nous amène à interroger les questions curriculaires et épistémologiques posées par une telle approche. L'introduction de cette discipline nouvelle, " sciences technologies société ", en remplacement des disciplines scientifiques traditionnelles (biologie, chimie, physique) a dû être abandonnée au profit de la poursuite d'un enseignement de contenus des disciplines scientifiques séparées. Pour Gaskell (2001), cette centration sur les contenus des disciplines scientifiques traditionnelles tient à ce que la perception de ce qui constitue des programmes scientifiques valables pour les étudiants, les enseignants et les parents dérive fortement de la définition des sciences proposée par les milieux scientifiques universitaires. Les éléments curriculaires des STS sont souvent perçus comme une perte de temps, aux dépens de l'apprentissage de notions utiles à la réussite dans les milieux scientifiques universitaires. Même les enseignants et enseignantes de sciences ont rarement accordé leur appui à ces initiatives. Outre leur formation personnelle et les relations qu'ils entretiennent avec les milieux universitaires, leur attitude reçoit l'appui des élèves et de leurs parents, qui ont tout intérêt à ce que les sciences proposées à l'école soient celles qui sont les mieux reconnues, qui ouvrent les portes des universités et augmentent les possibilités de carrière. Ainsi un enseignement scientifique pertinent est vu par nombre d'enseignants, de parents et d'élèves comme celui qui prépare naturellement à l'entrée dans les filières scientifiques de l'université (Gaskell, 2001).

Cette centration sur les contenus des disciplines scientifiques traditionnelles, plutôt que sur " des sciences sociales se faisant passer pour des sciences ", nous informe en outre de la nature épistémologique des sciences scolaires. D'une part, une caractéristique de l'enseignement des disciplines scientifiques est de s'extraire du contexte social, économique, culturel et pratique à l'origine de la production des savoirs. Les éléments curriculaires des STS vont à l'encontre d'un tel détachement traditionnel des problèmes sociaux des disciplines scientifiques et des scientifiques eux-mêmes, comme le souligne Gaskell (2001). D'autre part, l'organisation de disciplines scientifiques en enseignement de contenus factuels revient à présenter des résultats ayant statut de vérité. La perspective STS conduit au contraire à enseigner 
également les processus d'élaboration des savoirs et à considérer la dimension sociale de la validation des savoirs dans des communautés structurées par des projets. Une telle différence d'approche renvoie aux deux visions de la culture scientifique (Roberts, 2007) présentées plus haut et aux finalités des curriculums. Si l'on poursuit l'analyse en distinguant deux polarités, non nécessairement exclusives ${ }^{8}$, la question se pose soit de former de futurs spécialistes, par acculturation à une discipline scientifique pertinente en elle-même (au sens où un corpus de contenus scientifiques peut en lui-même fournir un but de formation), soit de former tous les jeunes dans le cadre de l'éducation obligatoire à comprendre les sciences, leur nature et leur rôle dans la société, les savoirs fournissant dans ce cas une médiation pour la communication et l'action. Comme le souligne Fourez (1997) à propos de la culture scientifique, le curriculum vise-t-il alors à faire voir aux élèves le monde tel que les scientifiques le voient ou bien à apprendre aux élèves à utiliser les sciences pour voir et comprendre leur monde ? Autrement dit, s'agit-il d'enseigner la biologie, la chimie, la physique ou d'apprendre aux jeunes à « négocier » avec leur monde? Pour Fourez (1997), une analyse idéologique des implications du choix d'une perspective ou d'une autre peut nous aider à mieux comprendre les réticences vis-à-vis des approches STS. Cet auteur s'interroge sur l'intérêt de réduire l'éducation scientifique à un enseignement de contenus disciplinaires : pour les scientifiques et pour ce qu'il nomme l'« establishment », une centration de l'enseignement sur des contenus disciplinaires renforce la vision du monde de la communauté scientifique et en fait la seule norme socialement acceptable ; elle augmente le recours aux experts dans des situations spécifiques, en " délégitimant " les préoccupations de la population au sens large, ce qui limite un partage du pouvoir lors de demandes de participation du public aux décisions en matière scientifique. "Ainsi à qui bénéficie le plus l'éducation scientifique : aux élèves, aux scientifiques, aux futurs employeurs des jeunes, à d'autres ${ }^{9}$ ? » (Fourez, 1997). Poser une telle question appelle à ouvrir la discussion sur cette analyse idéologique et à la prolonger prudemment, comme son auteur le souligne. Elle implique un débat sur le partage du pouvoir et la pratique de la démocratie, entre ce que Fourez nomme " une science élitiste pour les privilégiés " contre, en référence au projet Science for All de l'American Association for the Advancement of Science (1989), une « science pour tous ».

Dans le document intitulé Project 2061 de l'American Association for the Advancement of Science (1989), les sciences sont présentées comme une accumulation de thèmes (en technologie, sciences de l'information, ingénierie, sciences sociales, sciences de la santé, mathématiques, en plus des traditionnelles physique, chimie, biologie et géologie) et des considérations sociales, politiques, éthiques en sont exclues. Ainsi, pour des sociétés savantes telles que l'American Association for the Advancement of Science, promouvoir le développement de la culture scientifique et technique consiste-t-il en un effort pour que la science et l'activité scientifique soient perçues comme voie privilégiée pour penser et décider?

\section{IMPORTANCE ACCORDÉE À L'ENGAGEMENT SOCIAL ET CIVIQUE DANS LA CULTURE SCIENTIFIQUE}

Quant à l'importance accordée à l'engagement des élèves dans la société, la littérature est aussi riche de diverses approches. Pour certains, il s'agit de permettre aux individus de communiquer sur des thèmes scientifiques, de "fonctionner minimalement » (sic !) en société, comme consommateurs des sciences et des produits des sciences, ainsi que comme citoyens. Par exemple, les personnes cultivées 
scientifiquement peuvent apporter un plus grand soutien aux sciences elles-mêmes, en particulier via le financement public (Shortland, 1988) ou leur participation au secteur économique de la production technoscientifique industrielle (Walberg, 1983). Pour d'autres, les personnes cultivées scientifiquement peuvent « participer de façon complète dans la société » (Bussière, Knighton \& Pennock, 2007), s'engager dans des activités politiques, des processus de décisions collectives ou de changement social (Prewitt, 1983 ; Roth \& Barton, 2004). Dans cette perspective, le développement de la culture scientifique est considéré comme un fondement de la pratique de la démocratie dans nos sociétés (Désautels, 2002) et comme un élément pour favoriser la responsabilité civique. Ceci posé, il est possible d'appréhender la littérature à la recherche de la (ou des) signification(s) du terme « literacy » selon une perspective sémantique. Une personne dite « literate » peut être considérée comme " éduquée » (dans le sens d'avoir appris), compétente ou capable d'agir socialement (Roberts, 2007). La littératie pour Bailey (1998), à partir des métaphores de Scribner (1986), renvoie à plusieurs notions. Selon la métaphore de l'« adaptation ", développer une culture fonctionnelle pour agir dans une gamme de situations quotidiennes en société implique l'acceptation du statu quo social, c'est-à-dire de la société telle qu'elle est. Selon la métaphore du power, posséder la culture est un outil puissant, utilisé par les élites pour maintenir leur relative position de supériorité. Développer la culture est alors un moyen pour les pauvres ou les démunis politiquement d'affirmer leur place en société : une visée émancipatoire est ici centrale. Enfin la métaphore du "state of grace " souligne une tendance des sociétés à attribuer des vertus particulières aux personnes cultivées.

II est possible de rapprocher à des fins heuristiques ces différentes perspectives des deux visions de la culture scientifique dessinées par Roberts (2007) et précédemment présentées comme deux extrêmes d'un continuum d'approches. De façon schématique, dans la vision 1, il s'agit de viser l'insertion socioprofessionnelle des individus dans la société telle qu'elle est. Dans la vision 2, la culture scientifique est plutôt conçue comme l'opportunité de développer des compétences sociales pour une action et une réflexion critique hors de l'école. II s'agit de former à la prise de décisions, à l'action et à la reconstruction sociale critique par l'exercice de l'investigation scientifique et la pratique de la critique en relation avec des préoccupations sociales.

\section{DISCUSSION}

Nous en sommes ainsi conduite à interroger les justifications de la promotion de la culture scientifique, identifiées dans la littérature et précédemment présentées, en regard des orientations contemporaines du curriculum scientifique.

\section{La culture scientifique : nouvelle orientation ou justification du curriculum ?}

Le besoin de soutenir ou de développer la compétitivité dans une économie mondialisée a comme conséquence majeure d'orienter les objectifs du curriculum scientifique vers la production d'un nombre adéquat de personnels scientifiques bien qualifiés (élite scientifique et task force) et la génération d'une population "scientifiquement alphabétisée " (Jenkins, 2009, p. 81). La métaphore de l'enseignement scientifique comme un "pipeline, avec une fuite ", destiné à fournir la prochaine génération de scientifiques est ainsi largement rejetée en faveur de l'idée d'une édu- 
cation scientifique pour tous, qui donc ne peut être justifiée que si elle a une portée universelle plutôt que d'être réservée à la minorité des futurs scientifiques (Osborne \& Dillon, 2008, p. 7). Pour Jenkins, qui s'appuie sur une synthèse de documents institutionnels (rapports de gouvernements, de parlements, de commissions, textes d'institutions internationales, d'associations et de think tanks), ce quelque chose peut être décrit par la scientific literacy ou la « science pour l'éducation à la citoyenneté » (Jenkins, 2009, p. 82). Pour cette dernière, les finalités socio-éducatives s'inscrivent dans la lignée du mouvement « sciences technologies sociétés » (STS). Une telle perspective nous semble rejoindre les finalités dites démocratiques ou culturelles de développement de la culture scientifique.

On voit que le lien entre éducation scientifique et développement économique a focalisé l'attention des chercheurs et des décideurs politiques. Mais un tel lien n'est ni simple en soi, ni facile à établir sur le plan méthodologique, et il est de plus lié à la science considérée. Or ces limites ne sont pas mentionnées dans les documents institutionnels qui présentent le curriculum scientifique. Au contraire, le lien entre éducation scientifique et développement économique est posé comme une évidence, un vœu partagé et une base commune sur laquelle justifier une réforme de l'éducation scientifique (Jenkins, 2009, p. 81). Ceci n'a pas empêché certains chercheurs d'émettre des critiques. Pour Gatto (2005) par exemple, le lien entre éducation scientifique et développement économique met l'accent sur des buts utilitaires plutôt que sur un large développement intellectuel, social, personnel de l'individu. Ce lien établit aussi des critères pour évaluer l'éducation scientifique dans le cadre de la compétition économique internationale. Sur ce plan, différentes initiatives de réformes des curriculums scientifiques sont en cours dans plusieurs pays et les réponses sont largement similaires, alors même que l'histoire et les systèmes éducatifs de ces pays sont différents. Ces réponses sont caractérisées par leur nature systémique : réforme de tous les éléments clés qui influencent l'intérêt et les résultats des élèves en sciences (Jenkins, 2009, p. 81-82). L'enquête PISA qui permet d'évaluer les compétences des élèves en lecture, mathématiques et sciences a également soulevé des objections, qui ont porté sur l'approche statistique employée (Ginsburg, Cooke, Leinwand et al., 2005 ; Wu, 2005), sur les difficultés de traduction en plusieurs langues, sur la forme des tests (questions à choix multiples ou questions fermées) et sur les contextes qui sous-tendent les questions posées (Grisay \& Monseur, 2007). A été remise en question l'approche consistant à proposer aux élèves plusieurs items sur un même contexte et a donc été questionnée la familiarité des élèves avec ces contextes. Le contenu des items ou les matériaux textuels utilisés ont également été estimés problématiques (Hatzinikita, Dimopoulos \& Christidou, 2008 ; Neubrand, 2004). En outre, des recherches ont été effectuées pour réinterpréter des résultats (Ginsburg, Cooke, Leinwand et al., 2005), prolonger les analyses (Lie \& Linnakyla, 2004) ou en conduire de nouvelles afin de fournir des explications sur les différences de résultats entre pays ou entre établissements scolaires (Marks, 2006 ; Suchaut, Duru-Bellat \& Mons, 2005 ; Turmo, 2004).

Des recherches ont également été conduites afin d'identifier la perception des enseignants sur ces grandes enquêtes internationales. Celles-ci sont considérées comme des évaluations externes à visée de jugement et de contrôle, en contraste avec l'évaluation interne aux établissements, qui se présente elle-même comme un procédé destiné à améliorer l'enseignement et l'apprentissage (Livingston \& McCall, 2005). Des enseignants de sciences jugent par ailleurs de façon différenciée l'importance des compétences scientifiques composant la culture scientifique dans le cadre de l'enquête PISA (Pinto \& El Boudamoussi, 2009). Ils attachent peu d'importance à la maîtrise par les élèves de l'investigation scientifique, à l'interprétation de preuves scientifiques et ils concentrent leur attention sur les savoirs scientifiques. 
Les activités de classe que ces enseignants de sciences expérimentés développent privilégient l'apprentissage de la description, l'explication et la prédiction de phénomènes scientifiques. S'ils reconnaissent également d'autres exigences imposées par les tests PISA à leurs élèves ou leurs activités, comme la maîtrise de la compréhension de textes et l'usage de documents scientifiques ou d'extraits de presse, ils estiment qu'il s'agit de compétences que l'éducation scientifique développe de façon mineure.

Par ailleurs, ces critiques dans le domaine de l'éducation scientifique rejoignent celles de chercheurs en éducation soulignant la vision pragmatique et finalisée de l'éducation partagée par les organismes internationaux (Banque mondiale, Union européenne, OCDE), partisans du lien entre développement économique et formation des personnes «tout au long de la vie » (voir par exemple Moens, 2006). Les grandes enquêtes statistiques internationales font ainsi l'objet de vives critiques questionnant leur pertinence (Fensham \& Harlen, 1999 ; Simola, 2005) ou pointant l'effet performatif des indicateurs de performance sur les politiques éducatives nationales (Cussó, 2006 ; Goldstein, 2004 ; Mons, 2008).

\section{Argument démocratique de la culture scientifique : un défi pour l'éducation aux sciences}

Ainsi nous en venons à discuter de l'ambition démocratique du développement de la culture scientifique et technique. Cette ambition constitue selon nous un véritable défi pour la recherche en éducation aux sciences. Comment en effet cerner et décider des besoins sociaux en sciences (Kolstø, 2001) ? Qui peut le faire ? Les chercheurs en éducation aux sciences ou les spécialistes des questions sociales - comme le proposait dans un texte stimulant et polémique Fensham (2002) -, ou encore les scientifiques de la nature et du vivant qui développent également des discours sur la nécessité de promouvoir la culture scientifique à l'école et dans le « grand public »?

Par ailleurs, à l'instar de la science considérée comme une évidence dans la plupart des documents institutionnels analysés par Jenkins (2009), la société est souvent prise comme une donnée non problématique ou comme une entité naturalisée dans les recherches en éducation aux sciences visant à contribuer à une éducation à la citoyenneté (Albe, 2007 ; Davies, 2004). Une telle naturalisation de la société nous semble également à l'œuvre dans de nombreux documents et recherches sur la culture scientifique. Nous avons discuté plusieurs approches de la culture scientifique qui peuvent être distinguées en fonction de leurs finalités éducatives, de la nature des savoirs mobilisés pour fonder un curriculum scientifique (en vue de développer la culture scientifique des jeunes) et de l'importance accordée à l'engagement social des jeunes. À un extrême, l'accent est mis sur l'apprentissage de contenus scientifiques choisis en référence aux disciplines académiques traditionnelles (vision 1 proposée par Roberts, 2007), sans que la société dans laquelle viser l'insertion socioprofessionnelle des jeunes ne soit présentée ou discutée. À un autre extrême, la culture scientifique est associée à une visée de démocratisation des sciences et d'empowerment ${ }^{10}$ citoyen. Les savoirs scolaires sont définis en référence à des situations sociales dans lesquelles les sciences sont impliquées (vision 2 proposée par Roberts, 2007), en vue d'un engagement social et critique des élèves. La culture scientifique est destinée dans ce cas à permettre aux jeunes de se situer dans le monde et de participer à sa (re)configuration. II s'agit alors aussi d'une « alphabétisation politique » selon l'expression de Hodson (1999, p. 789). 


\section{La culture scientifique en tensions entre différentes polarités}

Ainsi est à nouveau ouvert le débat sur la visée de l'éducation scientifique, et ce débat est situé entre deux pôles : une reproduction sociale ou une émancipation sociale et politique. D'autres couples d'oppositions binaires peuvent également être identifiés dans les discours des promoteurs des différentes versions de la scientific literacy. Quant à la nature des savoirs mobilisés pour développer la culture scientifique, les deux visions proposées par Roberts (2007) décrivent une opposition entre d'un côté savoirs et concepts scientifiques (vision 1) et, de l'autre, savoirs et compétences utiles pour la vie sociale (vision 2). Sur un plan épistémologique, nous avons également discuté plus haut une opposition entre enseignement des sciences comme corpus de contenus factuels désocialisés ou comme activité culturelle. Du point de vue curriculaire, une tension peut également être identifiée pour le développement de la culture scientifique en disciplines scientifiques traditionnelles séparées, en référence aux disciplines académiques des sciences de la matière et du vivant (physique-chimie, biologie), versus activités éducatives aux formes nouvelles, du type enseignement " sciences technologies sociétés " ou " éducations à 》 (la citoyenneté, la santé, l'environnement et le développement durable).

Une telle analyse des débats sur la culture scientifique en tension entre des couples d'oppositions binaires renvoie en outre à des controverses plus larges qui se développent dans d'autres champs de recherche ; les recherches en éducation aux sciences sur la scientific literacy semblent d'ailleurs entretenir des rapports plus ou moins développés avec ces différents champs. Par exemple, la nature des savoirs mobilisés dans les deux visions de la scientific literacy (Roberts, 2007) peut être rapprochée des débats en sciences de l'éducation sur le couple ou l'opposition savoirs/compétences. Les visées et configurations curriculaires pour la culture scientifique renvoient en outre à des débats de sociologie du curriculum sur le couple ou l'opposition entre curriculums sériels et curriculums intégrés et leurs effets sociaux.

Par ailleurs, l'opposition dans la nature épistémologique des savoirs mobilisés dans les différentes approches de la culture scientifique peut être confrontée aux débats qualifiés de " guerres des sciences ${ }^{11}$ " entre épistémologie classique des sciences et études sociales sur les sciences (ou science studies). La recherche en éducation aux sciences semble ici entretenir des rapports plus étroits avec ces débats, bien qu'il soit délicat de tracer des proximités, voire des filiations, pour l'ensemble des recherches développées dans les mondes francophone et anglophone et publiées en anglais par des chercheurs non anglo-saxons. En ce qui concerne la seule didactique des sciences française, qui d'ailleurs portait l'appellation "épistémologie scolaire " au début de son développement (Astolfi \& Develay, 1992), une référence très répandue à une épistémologie classique d'inspiration bachelardienne ou popérienne peut être identifiée dans les recherches. Plus récemment, des recherches en éducation aux sciences ont été développées avec un ancrage fort dans le champ des science studies, où les sciences sont l'objet des sciences sociales : sociologie de la connaissance, histoire sociale et culturelle des sciences, anthropologie de laboratoire. Ces études sociales sur les sciences ont montré que la validité d'un énoncé scientifique se mesure à la stabilité de son acceptation par les autres chercheurs. Autrement dit, une connaissance est établie parce que les scientifiques ont travaillé à sa stabilisation, à sa mise en circulation, à la traduction des énoncés et à la mise en relation de mondes distincts. Différentes communautés structurent les savoirs qui les intéressent dans un réseau relationnel et sociopolitique particulier (théorie de l'acteur-réseau de Callon et Latour). La science est conçue dans ce cadre comme une pratique sociale, une activité culturelle, une insti- 
tution historiquement datée et conçue pour réaliser les projets d'un certain type de société. En outre, ces projets peuvent être des innovations sociotechniques. On dira donc que le complexe (techno)scientifique et le corps social se (re)définissent et se (re)construisent simultanément (Pestre, 1995). Une conséquence de ces études sociologiques est que l'idée de savoirs "socialement construits " s'est largement répandue et a suscité de vives polémiques, les travaux de la "nouvelle » sociologie des sciences ayant souvent défendu une thèse forte contre le scientisme ordinaire des savants qui prétendent que la science dit la vérité des choses. Au contraire, ces études sociologiques ont enrichi nos manières de voir les sciences en déplaçant notre regard d'une science centrée sur une question à une vision plus ample de pratiques hétérogènes impliquant des dynamiques dans l'ensemble du corps social.

On retrouve ainsi en éducation aux sciences l'opposition entre une appréhension de la science envisagée comme une unité, par une histoire internaliste des concepts et des méthodes, et une étude externaliste des sciences, considérées dans une diversité de pratiques. Cette opposition semble recouvrir celle identifiée à propos de la culture scientifique entre enseignement d'un corpus de savoirs désocialisés et acculturation à des pratiques sociales et culturelles.

\section{EN GUISE DE CONCLUSION}

Un tel panorama des recherches, interventions éducatives et grandes enquêtes internationales sur la culture scientifique nous conduit à nous interroger sur les conceptions de la société, de la citoyenneté et de la démocratie implicitement promues dans les nombreux discours concernés. Suivant Dupuy (2002) qui, en philosophie des sciences, mettait en lumière, à la suite d'Illich ${ }^{12}$, des effets contreproductifs des sciences et des techniques dans le domaine social, structurel ou symbolique, s'agit-il avec la culture scientifique et technique de favoriser ou d'accompagner, ou encore d'éclairer des dynamiques sociales ou l'ordre social ? Pour qui souhaite comprendre et contribuer à une éducation aux sciences plus avertie des enjeux de la société contemporaine (Larochelle \& Désautels, 2001, p. 41), il est fructueux et essentiel de procéder à une analyse sociopolitique. Un curriculum est en effet avant tout un objet politique, économique, institutionnel (Ross, 2000) avant d'être un objet didactique (Lebeaume, 2009). Ainsi nous ne pouvons que terminer cet article par une invitation à (ré)interroger les enjeux sociaux et politiques de la culture scientifique aujourd'hui, dans le cadre de la reconfiguration actuelle des systèmes éducatifs et de leur gouvernance. Quel projet scolaire d'instruction, d'éducation ou de formation en sciences et techniques des jeunes du $\mathrm{Xxl}^{\mathrm{e}}$ siècle ?

Virginie Albe virginie.albe@stef.ens-cachan.fr STEF, École normale supérieure de Cachan

\section{NOTES}

1 La tendance précédemment présentée de désengagement politique des citoyens vis-à-vis des grands enjeux de société peut être confrontée à d'autres analyses dans le champ de la sociologie. Certains ont par exemple proposé l'idée de « tournant participatif » pour désigner le développement et la multiont par exemple proposé l'idée de « tournant participatif " pour désigner le développement et la multi-
plication des formes de mobilisation sociale ou de participation des individus, sensibles notamment aux préoccupations environnementalistes et à la responsabilité envers les générations futures. Ces formes nouvelles d'activisme se distinguent de formes traditionnelles de mobilisation sociale, telles le syndicalisme ou le militantisme politique. La tendance contemporaine de désengagement politique 
des citoyens peut en outre être rapprochée de la ré-apparition récente de la thèse du " déficit » des individus, non plus perçu comme un déficit de connaissances, mais désormais comme un déficit d'engagement à propos de débats publics sur des développements technoscientifiques controversés.

2 Le champ francophone de la recherche en didactique des sciences est en anglais nommé science education research, le terme « didactic » étant en outre connoté péjorativement dans cette langue. Les titres des principales revues publiées en langue anglaise comportent ainsi les mots « science education ". Outre cette question de traduction, il est également possible de voir dans le choix de ce vocable une volonté de ne pas borner les questions de recherche à l'instruction, l'enseignement et vocable une volonte de ne pas borner les questions de recherche à l'instruction, l'enseignement et
l'apprentissage des sciences. C'est aussi cette intention de dépasser l'idée de transmission de connaissances qui est soulignée dans l'usage du terme "éducation aux sciences " dans le monde de la recherche francophone.

3 Traduction libre.

4 Les deux langues du programme PISA piloté par l'OCDE sont l'anglais et le français. Les choix de traduction en français du terme "scientific literacy » ont été précisés dans OCDE (2006).

5 Sept handbooks (éditions de 1994 et 1998 du International handbook of science education ; éditions de 1973, 1986, 1994 et 2001 du Handbook of research on teaching ; édition de 1992 du Handbook of research on curriculum), des actes de colloques ou symposiums internationaux (sur la recherche en éducation aux sciences en Europe et publié en 1977 par l'IPN, I'Institut für die Pädagogik der Naturwissenschaften de l'Université de Kiel, et le Conseil de l'Europe, ou par la Royal Swedish AcaNaturwissenschaften de l'Université de Kiel, et le Conseil de l'Europe, ou par la Royal Swedish Aca-
demy of Sciences en 1989 ; sur le concept de scientific literacy, en 1996, colloques ayant eu lieu à I'IPN et à Oslo ; les éditions de 1997, 1999, 2001 et 2003 des articles sélectionnés des conférences de l'European Science Education Research Association, de l'International Council of Associations for Science Education en 2000), des rapports ou fascicules de revues sur science literacy (le vol. 6 paru en 1997 d'Innovations in Science and Technology Education fondé par l'UNESCO ; les volumes Changing the subject, voir Black \& Atkin, 1996 pour l'OCDE et Bold ventures, vol 2. 'Case studies in US innovations in science education, voir Raizen \& Britton, 1997), des articles de recherche en éducation ( 15 issus de la base de données ERIC, Educational Resources Information Center, et 15 issus des actes de la conférence annuelle de la National Association for Research in Science Teaching en 2005).

6 Traduction libre de learned category.

7 Traduction libre de function in science category.

8 II serait en outre intéressant de s'interroger sur les croisements possibles entre ces deux polarités : comment par exemple forme-t-on les spécialistes à la communication et à l'action ? Comment, dans le cadre des participations de non-spécialistes à des débats publics sur des questions scientifiques (tel le débat national sur les nanosciences et les nanotechnologies durant l'hiver 2009-2010), interviennent dans les discussions ou prises de positions les connaissances en sciences et sur les sciences?

9 Traduction libre.

10 Nous retenons le terme anglais qui nous semble mieux à même de rendre compte de l'idée de nantir les personnes de "pouvoirs" (avec la présence du terme «power") que les traductions d'" empowerment » par « habilitation » par exemple.

11 J'ai déjà souligné par ailleurs (Albe, 2007, 2009) le caractère exagéré de l'usage du terme de “ guerre " à propos des controverses et polémiques sur la nature des sciences, au regard des effets des guerres véritables.

12 Illich (1971a) développa une critique radicale de la société techno-industrielle dans laquelle il considère les technologies comme une matrice qui façonne les rapports sociaux. Avec le concept de contre-productivité, Illich décrit comment les grandes institutions des sociétés industrielles modernes peuvent se constituer en obstacles à leur propre fonctionnement : par exemple, lorsque la médecine tue la maladie au détriment de la santé du patient (Illich, 1975), que les transports font perdre du temps (Illich, 1973a, 1973b), que l'école abêtit (Illich, 1971b), que les communications se développent au point que les personnes ne s'écoutent plus...

\section{BIBLIOGRAPHIE}

ALBE V. (2007). Des controverses scientifiques socialement vives en éducation aux sciences. Etat des recherches et perspectives. Mémoire de synthèse pour l'habilitation à diriger des recherches, université Lumière-Lyon 2.

ALBE V. (2008). «Culture scientifique et technique/Scientific and technological literacy : Quelles visées éducatives ? Quelles références pour les savoirs scolaires? " In C. Gardiès, I. Fabre, C. Ducamp \& V. Albe (dir.), Éducation à l'information et éducation aux sciences: quelles formes scolaires ? Toulouse : Éd. Cépaduès, p. 191-224.

ALBE V. (2009). Enseigner des controverses. Rennes : Presses universitaires de Rennes.

AMERICAN ASSOCIATION FOR THE ADVANCEMENT OF SCIENCE (1989). Project 2061: Science for all Americans. A project 2061 report on literacy goals in science, mathematics and technology. Washington: American Association for the Advancement of Science. 
AMERICAN ASSOCIATION FOR THE ADVANCEMENT OF SCIENCE (1993). Benchmarks for science literacy. Washington : American Association for the Advancement of Science.

ARONS A. (1983). «Achieving wider scientific literacy ». Dædalus, vol. 112, n 2, p. 91-122.

ASTOLFI J.-P. \& DEVELAY M. (1992). La didactique des sciences. Paris : PUF.

BAILEY P. (1998). "Conceptions of scientific literacy: Making sense of a proposed national science curriculum framework ". Alberta Science Education Journal, vol. 30, $\mathrm{n}^{\circ} 2$, p. 52-59.

BLACK P. \& ATKIN J. (1996). Changing the subject: Innovations in science, mathematics and technology education. Londres : Routledge.

BLADES D. (1997). Procedures of power and curriculum change: Foucault and the quest for possibilities in science education. New York: Peter Lang.

BRANSCOMB A. (1981). "Knowing how to know ». Science, Technology \& Human Values, vol. $6, n^{\circ} 36$, p. 5-9.

BUSSIĖRE P., KNIGHTON T. \& PENNOCK D. (2007). Measuring up: Canadian results of the OECD PISA study. The performance of Canada's youth in science, reading and mathematics. 2006 first results for Canadians aged 15. Canada : Minister of Industry. Disponible sur Internet à l'adresse : <http://www.pisa.gc.ca/eng/pdf/81-590-E.pdf> (consulté le 26 janvier 2011).

BYBEE R. (1991). "Science-technology-society in science curriculum: The policy-practice gap ». Theory into Practice, vol. 30, $\mathrm{n}^{\circ}$ 4, p. 294-302.

BYBEE R. (1997). Achieving scientific literacy: From purposes to practices. Portsmouth : Heinemann.

BYBEE R., McCRAE B. \& LAURIE R. (2009). "PISA 2006: An assessment of scientific literacy ". Journal of Research in Science Teaching, vol. 46, n 8, p. 865-883.

CARTER L. (2005). " Globalisation and science education: Rethinking science education reforms ". Journal of Research in Science Teaching, vol. 42, n 6, p. 561-580.

COMMISSION EUROPÉENNE (2004). Europe needs more scientists. Report by the high level group on increasing human resources for science and technology in Europe. Bruxelles: Commission européenne. Disponible sur Internet à l'adresse : <http://ec.europa.eu/ research/conferences/2004/sciprof/pdf/final_en.pdf> (consulté le 26 janvier 2011).

COMMISSION EUROPÉENNE (2007). Science education NOW: A renewed pedagogy for the future of Europe. Bruxelles : Commission européenne. Disponible sur Internet à l'adresse : <http://www.eurosfaire.prd.fr/7pc/bibliotheque/consulter. php?id=535> (consulté le 26 janvier 2011).

CUSSÓ R. (2006). "La politique des statistiques : l'influence des organisations internationales ». Communication présentée au colloque sur l'évaluation des politiques d'éducation et de formation, INRP, Lyon.

DAVIES I. (2004). "Science and citizenship education ". International Journal of Science Education, vol. 26, $\mathrm{n}^{\circ} 14$, p. 1751-1763.

DE BOER G. (2000). " Scientific literacy: Another look at historical and contemporary meanings and its relationship to science education reform ". Journal of Research in Science Teaching, vol. 37, n 6, p. 582-601.

DÉSAUTELS J. (2002). " L'alphabétisation technoscientifique et la démocratisation de la démocratie ". Revue canadienne de l'enseignement des sciences, des mathématiques et des technologies, vol. 2, $n^{\circ} 2$, p. 189-195.

DÉSAUTELS J. \& LAROCHELLE M. (2003). "Educación cientifica: el regresso del ciudadano y de la ciudadana ». Enseñanza de las Ciencas, vol. 21, n 1, p. 3-20.

DOW W. (2006). " The need to change pedagogies in science and technology subjects: A European perspective ". International Journal of Technology and Design Education, vol. 16, $n^{\circ} 3$, p. 307-321.

DRIVER R., LEACH J., MILLAR R. \& SCOTT P. (1996). Young people's image of science. Buckingham : Open University Press.

DUPUY J.-P. (2002). Pour un catastrophisme éclairé. Paris : Éd. du Seuil.

FENSHAM P. (1993). "Academic influence on school science curricula ». Journal of Curriculum Studies, vol. 25, n 1, p. 53-64.

FENSHAM P. (2002). " De nouveaux guides pour l'alphabétisation scientifique ». Revue canadienne de l'enseignement des sciences, des mathématiques et des technologies, vol. $2, n^{\circ} 2$, p. $133-150$. 
FENSHAM P. \& HARLEN W. (1999). «School science and public understanding of science ». International Journal of Science Education, vol. 21, n 7, p. 755-763.

FOUREZ G. (1997). "Scientific and technological literacy as a social practice ». Social Studies of Science, vol. 27, $n^{\circ}$ 6, p. 903-936.

GASKELL P. (1989). "Science and technology in British Columbia: A course in search of a community ». Pacific Education, vol. 1, n० 3 , p. 1-10.

GASKELL P. (2001). "STS in a time of economic change: What's love got to do with it? » Revue canadienne de l'enseignement des sciences, des mathématiques et des technologies, vol. $1, n^{\circ} 4$, p. 385-398.

GATTO J. (2005). Dumbing us down. The hidden curriculum of compulsory schooling. Gabriola Island: New Society Publishers.

GINSBURG A., COOKE G., LEINWAND S., NOELL J. \& POLLOCK E. (2005). Reassessing US international mathematics performance: New findings from the 2003 TIMSS and PISA. Washington : American Institutes for Research.

GOLDSTEIN H. (2004). " International comparisons of student attainment: Some issues arising from the PISA study ". Assessment in Education Principles, Policy and Practice, vol. 11, $n^{\circ} 3$, p. 319-330.

GRISAY A. \& MONSEUR C. (2007). "Measuring the equivalence of item difficulty in the various versions of an international test ». Studies in Educational Evaluation, vol. 33, $\mathrm{n}^{\circ} 1$, p. 69-86.

HATZINIKITA V., DIMOPOULOS K. \& CHRISTIDOU V. (2008). "PISA test items and school textbooks related to science: A textual comparison ». Science Education, vol. 92, $\mathrm{n}^{\circ} 4$, p. 664-687.

HODSON D. (1999). « Going beyond cultural pluralism: Science education for sociopolitical action ». Science Education, vol. 83, n 6, p. 775-796.

HODSON D. (2003). "Time for action: Science education for an alternative future ". International Journal of Science Education, vol. 25, n 6, p. 645-670.

HOLTON G. (1998). «1948: The new imperative for science literacy ». Journal of College Science Teaching, vol. 8, $\mathrm{n}^{\circ} 1$, p. 181-185.

HURD P. (1958). « Science literacy: Its meaning for American schools ». Educational Leadership, vol. 16, $\mathrm{n}^{\circ}$ 1, p. 13-16.

ILLICH I. (1971a). Libérer l'avenir. Paris : Éd. du Seuil.

ILLICH I. (1971b). Une société sans école. Paris : Éd. du Seuil.

ILLICH I. (1973a). Énergie et équité. Paris : Éd. du Seuil

ILLICH I. (1973b). La convivialité. Paris : Éd. du Seuil.

ILLICH I. (1975). Némésis médicale. Paris : Éd. du Seuil.

JENKINS E. (2009). «Reforming school science education: A commentary on selected reports and policy documents ». Studies in Science Education, vol. 45, n 1, p. 65-92.

KOBALLA T., KEMP A. \& EVANS R. (1997). « The spectrum of scientific literacy ». The Science Teacher, vol. 64, $\mathrm{n}^{\circ}$, p. 407-421.

KOLSTØ S. (2001). « Scientific literacy for citizenship: Tools for dealing with the science dimension of controversial socioscientific issues ». Science Education, vol. 85, $\mathrm{n}^{\circ} 3$, p. 291-310.

LAROCHELLE M. \& DÉSAUTELS J. (2001). « Les enjeux socioéthiques des désaccords entre scientifiques : un aperçu de la construction discursive d'étudiants et étudiantes ». Revue canadienne de l'enseignement des sciences, des mathématiques et des technologies, vol. $1, \mathrm{n}^{\circ} 1$, p. 39-60.

LAUGKSCH R. (2000). "Scientific literacy: A conceptual overview ». Science Education, vol. $84, \mathrm{n}^{\circ} 1$, p. $71-94$.

LEBEAUME J. (2009). “L'investigation dans l'enseignement des sciences : actualité des enjeux ". Communication présentée aux journées d'études S-TEAM, Grenoble. Disponible sur Internet à l'adresse : <http://iufm.ujf-grenoble.fr/index.php/accueil/188. html?start=5> (consulté le 26 janvier 2011).

LEGENDRE P. (2004). Ce que l'Occident ne voit pas de l'Occident. Paris : Éd. des Mille et une nuits.

LIE S. \& LINNAKYLA P. (2004). “ Nordic PISA 2000 in a sociocultural perspective ". Scandinavian Journal of Educational Research, vol. 48, n³, p. 227-230. 
LIVINGSTON K. \& MCCALL J. (2005). « Evaluation: Judgemental or developmental? 》 European Journal of Teacher Education, vol. 28, $n^{\circ} 2$, p. 165-178.

MARKS G. (2006). «Are between- and within-school differences in student performance largely due to socio-economic background? Evidence from 30 countries ». Educational Research, vol. 48, $\mathrm{n}^{\circ} 1$, p. 21-40.

MAYER V. \& KUMANO Y. (2002). « The philosophy of science and global science literacy ». In V. Mayer (dir.), Global science literacy. Dordrecht : Kluwer Academic Publishers.

MILLAR R. (1996). «Towards a science curriculum for public understanding ». School Science Review, vol. $77, \mathrm{n}^{\circ} 1, \mathrm{p} .7-18$.

MILLAR R. (2006). " Twenty first century science: Insights from the design and implementation of a scientific literacy approach in school science ». International Journal of Science Education, vol. 28, n¹3, p. 1499-1521.

MILLER J. (1983). « Scientific literacy: A conceptual and empirical review ». Dædalus, vol. $112, n^{\circ} 2$, p. 29-48.

MOENS F. (2006). «PISA : Controverses et débats en Belgique francophone. Des indicateurs à la qualité ? "Communication présentée au colloque sur l'évaluation des politiques d'éducation et de formation, INRP, Lyon.

MONS N. [coord.] (2008). Revue française de pédagogie, $\mathrm{n}^{\circ} 164$ : «Évaluation des politiques éducatives et comparaisons internationales ».

NEUBRAND M. (2004). "The PISA-study: Differentiated assessment of "mathematical literacy" ". In J. Emanuelsson \& D. Clarke (dir.), Contrasting comparative research on teaching and learning in mathematics. Cape Town : International Group for the Psychology of Mathematics Education, p. 26-30.

OCDE (2006). Assessing scientific, reading and mathematical literacy. A framework for PISA 2006. Paris : OCDE.

OSBORNE J. (2007). "Science education for the twenty first century ». Eurasia Journal of Mathematics, Science and Technology Education, vol. 3, $\mathrm{n}^{\circ}$ 3, p. 173-184.

OSBORNE J. \& DILLON J. (2008). Science education in Europe: Critical reflections. A report to the Nuffield Foundation. Londres : King's College Press.

PESTRE D. (1995). «Pour une histoire sociale et culturelle des sciences. Nouvelles définitions, nouveaux objets, nouvelles pratiques ". Annales. Histoire, sciences sociales, $\mathrm{n}^{\circ} 3$, p. 487-522.

PINTO R. \& EL BOUDAMOUSSI S. (2009). « Scientific processes in PISA tests observed for science teachers ». International Journal of Science Education, vol. $31, \mathrm{n}^{\circ} 16$, p. $2137-$ 2159.

PREWITT K. (1983). « Scientific illiteracy and democratic theory ». Dædalus, vol. 112, $\mathrm{n}^{\circ} 2$, p. 49-64.

RAIZEN S. \& BRITTON E. (1997). Bold ventures, vol. 2: Case studies in US innovations in science education. Dordrecht : Kluwer Academic Press.

ROBERTS D. (1983). Scientific literacy: Towards balance in setting goals for school science programs. Ottawa : Science Council of Canada.

ROBERTS D. (2007). «Scientific literacy/science literacy ». In S. Abell \& N. Lederman (dir.), Handbook of research on science education. Mahwah : Lawrence Erlbaum, p. 729-780.

ROBINE F. (2009). “L'enseignement des sciences, France et Europe ». Communication présentée aux journées d'études S-TEAM, Grenoble. Disponible sur Internet à l'adresse : <http://iufm.ujf-grenoble.fr/index.php/accueil/188.html?start=5> (consulté le 26 janvier 2011).

ROSS A. (2000). Curriculum, construction and critique. Londres : Falmer Press.

ROTH W. \& BARTON A. (2004). Rethinking scientific literacy. New York : Routledge Falmer.

SCRIBNER S. (1986). "Literacy in three metaphors ». In N. Stein (dir.), Literacy in American schools: Learning to read and write. Chicago : University of Chicago Press, p. 7-22.

SHAMOS M. (1995). The myth of scientific literacy. New Brunswick : Rutgers University Press.

SHEN B. (1975). "Science literacy and the public understanding of science ». In S. Day (dir.), Communication of scientific information. Bâle : Karger, p. 44-52.

SHORTLAND M. (1988). «Advocating science: Literacy and public understanding ». Impact of Science on Society, vol. 38, $\mathrm{n}^{\circ} 4$, p. 305-316.

SIMOLA H. (2005). "The Finnish miracle of PISA: Historical and sociological remarks on teaching and teacher education ». Comparative Education, vol. 41, n 4, p. 455-470. 
SNOW C. (1962). The two cultures and the scientific revolution. Cambridge : Cambridge University Press.

SOLOMON J. (1998). "The science curricula of Europe and the notion of scientific culture ". In D. Roberts \& L. Ostman (dir.), Problems of meaning in science curriculum. New York: Teachers College Press, p. 166-177.

SUCHAUT B., DURU-BELLAT M. \& MONS N. (2005). "Organisation scolaire et inégalités sociales de performance : les enseignements de l'enquête PISA ". Éducation et formations, $\mathrm{n}^{\circ} 70, \mathrm{p} .123-131$.

TURMO A. (2004). " Scientific literacy and socio-economic background among 15-year-olds. Nordic perspective ". Scandinavian Journal of Educational Research, vol. 48, $n^{\circ} 3$, p. 287-305.

WALBERG H. (1983). "Scientific literacy and economic productivity in international perspective ". Dædalus, vol. 112, $\mathrm{n}^{\circ}$ 2, p. 1-28.

WU M. (2005). "The role of plausible values in large-scale surveys ". Studies in Educational Evaluation, vol. 31, $\mathrm{n}^{\circ}$ 2, p. 114-128.

YAGER R. (1996). "History of science/technology/society as reform in the United States ». In R. Yager (dir.), STS as reform in science education. Albany : Suny Press, p. 3-15. 